TRANSACTIONS OF THE

AMERICAN MATHEMATICAL SOCIETY

Volume 184, October 1973

\title{
ANALYTIC FUNCTIONS CHARACTERIZED BY THEIR MEANS ON AN ARC
}

\author{
BY \\ CHIN.HUNG CHING AND CHARLES K. CHUI
}

ABSTRACT. It is known that a function $f$, holomorphic in the open unit disc $U$ with $C^{1+\epsilon}$ boundary data for some $\epsilon>0$, is uniquely determined by its arithmetic means over equally spaced points on $\partial U$. By using different techniques, we weaken the hypothesis $C^{1+\epsilon}(\partial U)$ to functions with $L^{p}$ derivatives, $1<p \leq \infty$. We also prove that a function is determined by its averages over an arc $K$ if $\bar{f}$ is holomorphic in a neighborhood of $\bar{U}$, and that this result is false for some functions $f$ in $A \cap C^{\infty}(\bar{U})$. On the other hand, we can capture a $A \cap C^{2}(\bar{U})$ function from its means and shifted means on $K$.

1. Introduction and results. Let $U$ denote the open unit disc in the complex plane with closure $\bar{U}$ and boundary $T$. As usual, let $H^{p}$ be the Hardy spaces and $A$ be the space of functions holomorphic in $U$ and continuous in $\bar{U}$. For a continuous function $f$ on an arc $\left\{e^{i 2 \pi t}: t_{1} \leq t \leq t_{2}\right\}$ of $T$, we consider its arithmetic means

$$
s_{n}\left(f ; t_{1}, t_{2}\right)=\frac{1}{n} \sum_{k=1}^{n} f\left(\exp \left(i 2 \pi k\left(t_{2}-t_{1}\right) / n+i 2 \pi t_{1}\right)\right)
$$

and

$\tilde{s}_{n}\left(f ; t_{1}, t_{2}\right)=\frac{f\left(\exp \left(i 2 \pi t_{1}\right)\right)+f\left(\exp \left(i 2 \pi t_{2}\right)\right)}{2 n}+\frac{1}{n} \sum_{k=1}^{n-1} f\left(\exp \left(i 2 \pi k\left(t_{2}-t_{1}\right) / n+i 2 \pi t_{1}\right)\right)$, $n=1,2, \ldots$. Then $s_{n}(f ; 0,1)=\tilde{s}_{n}(f ; 0,1)$ for all $n$. The following result is proved in [1].

Theorem A. Let $f(z)=\Sigma a_{n} z^{n}$, where $a_{n}=O\left(1 / n^{1+\epsilon}\right)$ for some $\epsilon>0$, be such that $s_{n}(f ; 0,1)=0$ for every $n=1,2, \ldots$. Then $f$ is the zero function.

In particular, if $f \in C^{1+\epsilon}(\bar{U})$, then $f$ is uniquely determined by the $s_{n}(f ; 0,1)$. It is pointed out in [1] that there exist polynomials $p_{m}$ with $s_{n}\left(p_{m} ; 0,1\right)=\delta_{n, m}$ for all $m$ and $n$. In this paper, by using techniques different from those used in [1], we obtain

Received by the editors August 8, 1972 and, in revised form, January 19, 1973. AMS (MOS) subject classifications (1970). Primary 30A82.

Key words and phrases. Arithmetic means on an arc, Möbius function, holomorphic functions, uniqueness and nonuniqueness, representation formulas. 
Theorem 1. Let $f$ be a function bolomorpbic in $U$ with $f^{\prime} \in H^{p}, 1<p \leq \infty$, and $s_{n}(f ; 0,1)=0$ for each $n=1,2, \ldots$. Then $f$ is the zero function.

Hence, if $f \in C^{1}(\bar{U}), f$ is uniquely determined by the means $s_{n}(f ; 0,1)$.

Since each $f \in A$ is determined by its values on an arc $T$, it is natural to ask (cf. [3]) for a given $\delta, 0<\delta<1$, whether $s_{n}(f ; 0, \delta)=0$ or $\tilde{s}_{n}(f ; 0, \delta)=0$, $n=1,2, \ldots$, would imply that $f$ is the zero function or not. We answer this question in the following theorems.

Theorem 2. For each $\delta$ with $0<\delta<1$, there exists a nonzero function $f \in A$, infinitely differentiable relative to $\bar{U}$, sucb that $s_{n}(f ; 0, \delta)=\tilde{s}_{n}(f ; 0, \delta)=0$ for all $n=1,2, \ldots$.

On the other hand, we have

Theorem 3. Let $0<\delta<1$ and $f$ be bolomorpbic in a neighborbood of the closed disc $\bar{U}$. Then $f$ is the zero function if either

(i) $\tilde{s}_{n}(f ; 0, \delta)=0, n=1,2, \ldots$, or

(ii) $s_{n}(f ; 0, \delta)=0, n=1,2, \cdots$, is satisfied.

Since $f \in C^{\infty}(\bar{U})$ is not, in general, determined by its means $s_{n}$ or $\tilde{s}_{n}$ on a proper subarc $\left\{e^{i 2 \pi t}: 0 \leq t \leq \delta\right\}, 0<\delta<1$, of $T$, we also consider the shifted means

$$
t_{n}(f ; 0, \delta)=\frac{f\left(e^{i 2 \pi \delta}\right)}{2 n}+\frac{1}{n} \sum_{k=1}^{n-1} f\left(e^{i 2 \pi(2 k-1) \delta /(2 n-1)}\right)
$$

and have

Theorem 4. Let $f \in A$ with $f^{\prime}\left(e^{i 2 \pi t}\right) \in L^{p}(0, \delta)$ for some $p, 1<p \leq \infty$, where $0<\delta<1$, such that either

(i) $\tilde{s}_{n}(f ; 0, \delta)=t_{n}(f ; 0, \delta)=0, n=1,2, \ldots$, or

(ii) $s_{n}(f ; 0, \delta)=t_{n}(f ; 0, \delta)=0, n=1,2, \ldots$, is satisfied. Then $f$ is the zero function.

Combining Theorems 3 and 4 and results from [2] and [6], we can give a representation formula of a function in terms of its means and shifted means on an arc.

2. Proof and consequences of Theorem 1. We now prove Theorem 1. It is clear that $f^{\prime} \in H^{p}$ implies $f \in A$. Without loss of generality, we assume $1<p \leq 2$. For $j=1,2, \cdots$, we define the functions

$$
\phi_{j}(t)= \begin{cases}-j t+[j t]+1 / 2 & \text { for } t \neq k / j, k=0, \pm 1, \ldots, \\ 0 & \text { otherwise, }\end{cases}
$$


where $[x]$, as usual, denotes the largest integer no greater than $x$. Since $s_{j}(f ; 0,1)=0$, we have

$$
\int_{0}^{1} f\left(e^{i 2 \pi t}\right) d t=0
$$

Hence, for all $j$,

$$
\int_{0}^{1} \phi_{j}(t) d f\left(e^{i 2 \pi t}\right)=-j\left\{\tilde{s}_{j}(f ; 0,1)-\int_{0}^{1} f\left(e^{i 2 \pi t}\right) d t\right\}=0 .
$$

Let $\mu(n)$ be the Möbius function, that is,

$$
\begin{aligned}
\mu(n) & =1 \quad \text { if } n=1, \\
& =(-1)^{k} \text { if } n=p_{1} \cdots p_{k} \text { with distinct primes } p_{j}, \\
& =0 \quad \text { if } p^{2} \mid n \text { for some } p>1 .
\end{aligned}
$$

Since

$$
\phi_{j}(t)=\sum_{k=1}^{\infty} \frac{\sin 2 \pi k j t}{k \pi},
$$

it is easy to verify that, for all $n$ and $N$,

$$
\pi \sum_{k=1}^{N} \mu(k) \frac{\phi_{k n}(t)}{k}=\sin 2 \pi n t+\sum_{k=N+1}^{\infty}\left\{\sum_{j \mid k}^{i \leq N} \mu(j)\right\} \frac{\sin 2 \pi k n t}{k},
$$

where the series converges for all $t$. By (2) and (3), we have

$$
\begin{gathered}
\int_{0}^{1} \sin 2 \pi n t d f\left(e^{i 2 \pi t}\right)=\int_{0}^{1}\left\{\sin 2 \pi n t-\pi \sum_{k=1}^{N} \mu(k) \frac{\phi_{k n}(t)}{k}\right\} d f\left(e^{i 2 \pi t}\right) \\
=-i 2 \pi \int_{0}^{1} \sum_{k=N+1}^{\infty}\left\{\sum_{j \mid k}^{j \leq N} \mu(j)\right\} \frac{\sin 2 \pi k n t}{k} f^{\prime *}\left(e^{i 2 \pi t}\right) e^{i 2 \pi t} d t
\end{gathered}
$$

where $f^{\prime *}$ is the a.e. radial limit of $f^{\prime}$ and we have used the standard technique of passing to the radial limit. By applying the Hölder inequality and the HardyLittlewood inequality for $L^{q}, 1 / p+1 / q=1$ (cf. [7, p. 110]), we obtain

$$
\begin{aligned}
& \left|\int_{0}^{1} \sin 2 \pi n t d f\left(e^{i 2 \pi t}\right)\right| \leq 2 \pi\left\|f^{\prime}\right\|_{p}\left\|\sum_{N+1}^{\infty}\left\{\sum_{j \mid k}^{j \leq N} \mu(j)\right\} \frac{\sin 2 \pi k n t}{k}\right\|_{q} \\
& \leq C_{f}\left\{\left.\left.\sum_{k=N+1}^{\infty} k^{q-2} \frac{1}{k^{q}}\right|_{j \mid k} ^{j \leq N} \mu(j)\right|^{q}\right\}^{1 / q} \leq C_{f}\left\{\sum_{k=N+1}^{\infty} \frac{[d(k)]^{q}}{k^{2}}\right\}^{1 / q}
\end{aligned}
$$

where $d(k)$ is the number of divisors of $k$. Since $d(k)=O\left(k^{\delta}\right)$ for any $\delta>0$ (cf. $[4$, p. 260]), we can conclude, by letting $N$ tend to infinity, that 


$$
\int_{0}^{1} \sin 2 \pi n t d f\left(e^{i 2 \pi t}\right)=0
$$

for $n=1,2, \ldots$. Thus, by combining (1) and (4), we have

$$
\int_{T} \bar{z}^{n} f(z) d z=\int_{T}\left(z^{n}+\bar{z}^{n}\right) f(z) d z=0
$$

for $n=0,1, \cdots$, and hence $f$ is the zero function.

In the above proof, the number theory technique we used is elementary. We remark, however, that if we use a much deeper result of $\mathrm{H}$. Davenport, we can push Theorem 1 to hold for $p=1$. Also, we have actually proved the following

Corollary 1. Let $F(t)$ be an even function continuous on $[-\delta, \delta]$ such that $F^{\prime}(t) \in L^{p}(-\delta, \delta)$ for some $p, 1<p \leq \infty$, and such that

$$
\tilde{s}_{n}(F)=\frac{1}{n} \sum_{k=1}^{n} F\left(\frac{2 k \delta}{n}-\delta\right)=0
$$

for $n=1,2, \ldots$ Then $F=0$.

It is natural to ask whether we can move the roots of unity, so that the means of $f$ on $T$ can still uniquely determine $f$. Theorem 1 implies that we can use the conformal images of the roots of unity under a linear fractional transformation. We also have

Corollary 2. Let $\rho$ be a $C^{1}$ diffeomorpbism of $T$ with $\rho(\bar{z})=\overline{\rho(z)}$ for $z \in T$. Let $z_{n, k}=\rho\left(e^{i 2 \pi k / n}\right), k=1, \cdots, n$. If $f$ is bolomorpbic in $U$ with $f^{\prime} \in H^{p}, 1<$ $p \leq \infty$, and satisfies $(1 / n) \Sigma_{k=1}^{n} f\left(z_{n, k}\right)=0$ for $n=1,2, \ldots$. Then $f$ is the zero function.

To prove this, we let $g=f \circ \rho$ on $T$. Then by Corollary 1, we can conclude that $g\left(e^{i t}\right)=-g\left(e^{-i t}\right)$ for all $t$; and hence, for $z=\rho\left(e^{i t}\right)$ on $T$, we have $f(z)=$ $-f(\bar{z})$. That is, the function $f(z)+f(\bar{z})$, harmonic in $U$ and continuous on $\bar{U}$, is zero on $T$, and must be zero on $\bar{U}$. By the Cauchy-Riemann equations, $f=0$.

3. Proof of Theorem 2. Let $0<\delta<1$. To construct $f$, we let

$$
b(t)=\sum_{n=0}^{\infty} a_{n} \cos 2 \pi n t
$$

be a real-valued infinitely differentiable even function, not identically zero, with period one, such that $b(t)=0$ for $|t| \leq \delta / 2$ and $b(1)=0$. Let $b=\sum_{n=0}^{\infty} a_{n} \sin \pi n \delta$. If $b=0$, we define $g(t)=b(t)$. If $b \neq 0$, we define $g(t)=b^{2}(t)+c h(t)$ with

$$
c=-\frac{1}{b} \sum_{n=0}^{\infty} \sin \pi n \delta \int_{0}^{1} b^{2}(t) \cos 2 \pi n t d t .
$$


Then

$$
g(t)=\sum_{n=0}^{\infty} b_{n} \cos 2 \pi n t
$$

is a real-valued infinitely differentiable nonzero even function with period one and

$$
\sum_{n=1}^{\infty} b_{n} \sin \pi n \delta=0 \text {. }
$$

Now let

$$
f(z)=\sum_{n=0}^{\infty} b_{n} e^{-i \pi \delta n} z^{n}
$$

Since $b_{n}=O\left(1 / n^{k}\right)$ for any integer $k, f$ is holomorphic in $U$ and infinitely differentiable relative to $\bar{U}$. Furthermore, since $g(\delta / 2)=0$, we have, by $(G)$,

$$
f(1)=\sum_{n=0}^{\infty} b_{n} \cos \pi n \delta-i \sum_{n=0}^{\infty} b_{n} \sin \pi n \delta=g(\delta / 2)-i \sum_{n=0}^{\infty} b_{n} \sin \pi n \delta=0
$$

and

$$
f\left(e^{i 2 \pi \delta}\right)=g(\delta / 2)+i \sum_{n=0}^{\infty} b_{n} \sin \pi n \delta=0
$$

We can write

$$
f\left(e^{i 2 \pi t}\right)=\sum_{n=0}^{\infty} c_{n} \cos \frac{2 \pi n}{\delta}\left(t-\frac{\delta}{2}\right)+\sum_{n=0}^{\infty} d_{n} \sin \frac{2 \pi n}{\delta}\left(t-\frac{\delta}{2}\right)
$$

for $0 \leq t \leq \delta$. Since $g(t)=0$ for $|t| \leq \delta / 2$, we can conclude from (5) and (7) that

$$
\begin{aligned}
f\left(e^{i 2 \pi t}\right) & =1 / 2\left\{f\left(e^{i 2 \pi t}\right)+f\left(e^{i 2 \pi(\delta-t)}\right)\right\}+\sum_{n=0}^{\infty} d_{n} \sin \frac{2 \pi n}{\delta}\left(t-\frac{\delta}{2}\right) \\
& =g\left(t-\frac{\delta}{2}\right)+\sum_{n=0}^{\infty} d_{n} \sin \frac{2 \pi n}{\delta}\left(t-\frac{\delta}{2}\right)=\sum_{n=0}^{\infty} d_{n} \sin \frac{2 \pi n}{\delta}\left(t-\frac{\delta}{2}\right)
\end{aligned}
$$

for $0 \leq t \leq \delta$. Thus, for all $m=1,2, \cdots$, we have

$$
\begin{aligned}
s_{m}(f ; 0, \delta) & =\tilde{s}_{m}(f ; 0, \delta)=\frac{1}{m} \sum_{k=1}^{m} f\left(e^{i 2 \pi k \delta / m}\right) \\
& =\frac{1}{m} \sum_{n=0}^{\infty} d_{n} \sum_{k=1}^{m} \sin 2 \pi n\left(\frac{k}{m}-\frac{1}{2}\right)=0 .
\end{aligned}
$$

We remark that our function $f$ can be made to be holomorphic in a neighborhood of the arc $\left\{e^{i 2 \pi t}: 0 \leq t \leq \delta\right\}$ by using Schwarz's reflection principle. 
4. Proof of Theorem 3. Let $f$ be holomorphic in the neighborhood $|z|<1 / r$, $0<r<1$, of $\bar{U}, 0<\delta<1$ and $\tilde{s}_{n}(f ; 0, \delta)=0, n=1,2, \ldots$. Define $g\left(e^{i 2 \pi t}\right)=$ $f\left(e^{i 2 \pi(t+\delta / 2)}\right)+f\left(e^{i 2 \pi(\delta / 2-t)}\right)$. Then $\tilde{s}_{n}(g ;-\delta / 2, \delta / 2)=2 \tilde{s}_{n}(f ; 0, \delta)=0$ for all $n$. Hence, by Corollary 1 , we have $f\left(e^{i 2 \pi(t+\delta / 2)}\right)=-f\left(e^{i 2 \pi(\delta / 2-t)}\right)$ for $|t| \leq \delta / 2$. Let $F(z)=f\left(e^{i \pi \delta} z\right)=\Sigma_{n=0}^{\infty} a_{n} z^{n}$. Then $F\left(e^{i 2 \pi t}\right)=-F\left(e^{-i 2 \pi t}\right)$ or

$$
\sum_{n=0}^{\infty} a_{n} \cos 2 \pi n t=0
$$

for $|t| \leq \delta / 2$. Since $F$ is holomorphic in $|z|<1 / r$ where $0<r<1$, we have $\left|a_{n}\right| \leq C_{r^{n}}$ for all $n$ and some $C$. From the estimate $|\cos n(x+i y)| \leq e^{n|y|}$, we see that the series $\Sigma a_{n} \cos n z$ converges uniformly on the strip $|\operatorname{Im} z| \leq \epsilon$ for $0<\epsilon<-\log r$. In virtue of $(8)$ and the identity theorem, we conclude that the function $\sum a_{n} \cos n z$ vanishes on the whole strip $|\operatorname{Im} z|<-\log r$, and hence, $f$ is the zero function. This proves the first part of the theorem.

Now suppose that $s_{n}(f ; 0, \delta)=0, n=1,2, \ldots$ We let $c=\left(f(1)-f\left(e^{i 2 \pi \delta}\right)\right) / 2$. Then $\tilde{s}_{n}(f ; 0, \delta)=s_{n}(f ; 0, \delta)+c / n=c / n$ for $n=1,2, \ldots$. By the first part of the theorem, it is sufficient to prove that $c=0$. To do this, we note that, since $\tilde{s}_{n}(f ; 0, \delta) \rightarrow 0$,

(9)

$$
\tilde{s}_{n}(f ; 0, \delta)=\frac{1}{n} \int_{0}^{1} f\left(e^{i 2 \pi \delta t}\right) d \phi_{n}(t)
$$

$$
=\frac{-i 2 \pi \delta}{n} \int_{0}^{1} \phi_{n}(t) f^{\prime}\left(e^{i 2 \pi \delta t}\right) e^{i 2 \pi \delta t} d t .
$$

Or, $c=\int_{0}^{1} \phi_{n} g$ where $g(t)$ is continuous on $[0,1]$. Since $\left|\phi_{n}(t)\right| \leq 1 / 2$ for all $t$ and all $n$, and since $\left|\int_{a}^{b} \phi_{n}(t) d t\right| \leq 1 / 4 n$ for all $n$ and $a, b$ with $0 \leq a \leq b \leq 1$, we can approximate $g$ in $L^{1}[0,1]$ by step functions and conclude, as in proving the Riemann-Lebesgue lemma, that $\int_{0}^{1} \phi_{n} g \rightarrow 0$. Hence, $c=0$. This completes the proof of the theorem.

5. Proof of Theorem 4 and a representation formula. Suppose that $f \in A$, $f^{\prime}\left(e^{i 2 \pi t}\right) \in L^{p}(0, \delta), 0<\delta<1$ and $1<p \leq \infty$. Let

$$
\begin{array}{rlrl}
g\left(e^{i 2 \pi t)}\right) & =f\left(e^{i 2 \pi t}\right), & & 0 \leq t \leq \delta, \\
& =f\left(e^{-i 2 \pi t}\right), & -\delta \leq t \leq 0 .
\end{array}
$$

It can be shown that, for each $m=1,2, \cdots$,

$$
\tilde{s}_{2 m-1}(g ;-\delta, \delta)=(2 m /(2 m-1)) t_{m}(f ; 0, \delta)
$$

and

$$
\tilde{s}_{2 m}(g ;-\delta, \delta)=\tilde{s}_{m}(f ; 0, \delta) .
$$

Hence, if $t_{m}(f ; 0, \delta)=\tilde{s}_{m}(f ; 0, \delta)=0$ for $m=1,2, \ldots$, then $\tilde{s}_{n}(g ;-\delta, \delta)=0$ 
for $n=1,2, \ldots$, so that $g=0$ by Corollary 1 . Or, $f$ vanishes on the arc $\left\{e^{i 2 \pi t}\right.$ : $0 \leq t \leq \delta\}$ and must be the zero function. Suppose $t_{m}(f ; 0, \delta)=s_{m}(f ; 0, \delta)=0$ instead. We then have, by $(10)$ and $(11)$, that $\tilde{s}_{2 m-1}(g ;-\delta, \delta)=0$ and $\tilde{s}_{2 m}(g ;-\delta, \delta)=\left(f(1)-f\left(e^{i 2 \pi \delta}\right)\right) / 4 m=c / 2 m$ for $m=1,2, \ldots$. Following the proof of Theorem 1, we have

$$
\int_{-\delta}^{\delta} \sin \left(\frac{2 \pi n t}{\delta}\right) d g\left(e^{i 2 \pi t}\right)=\pi \sum_{k=1}^{2 N} \frac{\mu(k)}{k} \int_{-\delta}^{\delta} \phi_{k n}\left(\frac{t}{\delta}\right) d g\left(e^{i 2 \pi t}\right)+o(1)
$$

where $o(1)$ tends to zero as $N$ approaches infinity uniformly in $n$. Thus, for odd $n$, we have

$$
\int_{-\delta}^{\delta} \sin \left(\frac{2 \pi n t}{\delta}\right) d g\left(e^{i 2 \pi t}\right)=\pi c \sum_{k=1}^{N} \frac{\mu(2 k)}{2 k}+o(1)=\frac{\pi c}{2} \mu(2) \sum_{k=1}^{N} \frac{\mu(k)}{k}+o(1) .
$$

We use the easy estimate. (cf. $\left[5\right.$, p. 335]) $\left|\sum_{k=1}^{N} \mu(k) / k\right| \leq 1$ to conclude that, for $n=1,2, \cdots$,

$$
\int_{-\delta}^{\delta} \sin \left(\frac{2 \pi n t}{\delta}\right) d g\left(e^{i 2 \pi t}\right)=a
$$

for some constant $a$ independent of $n$. Thus, $a=0$ by taking $n$ to infinity. That is, we have

$$
\int_{-\delta}^{\delta} g\left(e^{i 2 \pi t}\right) \cos \left(\frac{2 \pi n t}{\delta}\right) d t=0
$$

for all odd $n$. A similar argument shows that (12) holds for all even $n$. Thus, $g=$ 0 ; or $f$ is the zero function. This completes the proof of the theorem.

As mentioned in $\S 1$, we can reconstruct certain functions from their means on an arc. For example, we consider the trigonometric polynomials

$$
p_{n}\left(e^{i 2 \pi t}\right)=\sum_{k \mid n} \mu\left(\frac{n}{k}\right) \cos 2 \pi k t
$$

which have the property

$$
s_{m}\left(p_{n} ; 0,1\right)=\tilde{s}_{m}\left(p_{n} ; 0,1\right)=\delta_{m, n}
$$

(cf. [2, Lemma 1]). Let $0<\delta<1$. For $\lambda>0$, we consider the functions

$$
b_{\lambda}(z)=\exp \left\{\frac{-\log (1+\lambda)}{4 \pi} \int_{0}^{2 \pi \delta} \frac{e^{i t}+z}{e^{i t}-z} d t\right\} .
$$

If $k(t)$ is a continuous function on the arc $K=\left\{e^{i 2 \pi t}: 0 \leq t \leq \delta\right\}$, we denote $\tilde{s}_{\infty}(k ; 0, \delta)=\lim _{n \rightarrow \infty} \tilde{s}_{n}(k ; 0, \delta)$.

Now, let $f \in A \cap C^{2}(T)$. We first prove that 


$$
\tilde{s}_{m}(f ; 0, \delta)-\tilde{s}_{\infty}(f ; 0, \delta)=O\left(1 / m^{2}\right)
$$

and

$$
(2 m /(2 m-1))_{m}(f ; 0, \delta)-\tilde{s}_{\infty}(f ; 0, \delta)=O\left(1 / m^{2}\right) .
$$

To prove (14) we let $\phi_{m}(t)$ be as defined in $\$ 2$ and let $\psi_{m}(x)=\int_{0}^{x} \phi_{m}(t) d t$. Then it can be shown that $\psi_{m}(1)=\psi_{m}(0)=0$ and $0 \leq \psi_{m}(x) \leq 1 / 2 m$ for all $x$ and all $m$. Hence,

$$
\begin{aligned}
\tilde{s}_{m}(f ; 0, \delta) & -\tilde{s}_{\infty}(f ; 0, \delta)=\frac{1}{m} \int_{0}^{1} f\left(e^{i 2 \pi \delta t}\right) d \phi_{m}(t) \\
& =\frac{-i 2 \pi \delta}{m} \int_{0}^{1} \psi_{m}^{\prime}(t) f^{\prime}\left(e^{i 2 \pi \delta t}\right) e^{i 2 \pi \delta t} d t \\
& =\frac{i 2 \pi \delta}{m} \int_{0}^{1} \psi_{m}(t)\left[f^{\prime}\left(e^{i 2 \pi \delta t}\right) e^{i 2 \pi \delta t}\right]^{\prime} d t=O\left(\frac{1}{m^{2}}\right) .
\end{aligned}
$$

To prove (15), we define our functions $\eta_{n}(t)$ on $[0,1]$ as follows: For each $n$, $\eta_{n}(t)$ is piecewise linear with each slope equal to $-(2 n-1) / 2$; the only discontinuities of $\eta_{n}(t)$ are at $1 /(2 n-1), 3 /(2 n-1), \ldots, 1$, having a unit jump at $1 /(2 n-1), \ldots,(2 n-3) /(2 n-1)$ and a jump of value $1 / 2$ at 1 ; and finally,

$$
\eta_{n}(0)=\eta_{n}(1 /(2 n-1))=\cdots=\eta_{n}((2 n-3) /(2 n-1))=\eta_{n}(1)=0 .
$$

Hence, $\left|\eta_{n}(t)\right| \leq 1 / 2$ and $\eta_{n}(t)$ looks like a "sawtooth" function. Let $\xi_{n}(x)=$ $\int_{0}^{x} \eta_{n}(t) d t$. Then $\xi_{n}(0)=0, \xi_{n}(1)=-1 /(8 n-4)$ and $\left|\xi_{n}(x)\right| \leq 1 /(8 n-4)$ for all $x$ on $[0,1]$. It also follows that

$$
\begin{aligned}
\frac{2 m}{2 m-1} & t_{m}(f ; 0, \delta)-\tilde{s}_{\infty}(f ; 0, \delta)=\frac{2}{2 m-1} \int_{0}^{1} f\left(e^{i 2 \pi \delta t}\right) d \eta_{m}(t) \\
& =\frac{-i 4 \pi \delta}{2 m-1} \int_{0}^{1} \xi_{m}^{\prime}(t) f^{\prime}\left(e^{i 2 \pi \delta t}\right) e^{i 2 \pi \delta t} d t \\
& =\frac{i 4 \pi \delta}{2 m-1}\left\{\frac{f^{\prime}\left(e^{i 2 \pi \delta}\right) e^{i 2 \pi \delta}}{8 m-4}+\int_{0}^{1} \xi_{m}(t)\left[f^{\prime}\left(e^{i 2 \pi \delta t}\right) e^{i 2 \pi \delta}\right]^{\prime} d t\right\}=O\left(\frac{1}{m^{2}}\right),
\end{aligned}
$$

proving (15). Hence, by using (14) and (15) and a result in [2, Theorem 2], we see that the series

$$
\sum_{m=1}^{\infty}\left\{\tilde{s}_{m}(f ; 0, \delta)-\tilde{s}_{\infty}(f ; 0, \delta)\right\}_{2 m}\left(e^{i \pi(t / \delta-1)}\right)
$$

$$
+\sum_{m=1}^{\infty}\left\{\frac{2 m}{2 m-1} t_{m}(f ; 0, \delta)-\tilde{s}_{\infty}(f ; 0, \delta)\right\} p_{2 m-1}\left(e^{i \pi(t / \delta-1)}\right)+\tilde{s}_{\infty}(f ; 0, \delta)
$$


converges uniformly on $[-\delta, \delta]$ to a function $G(t)=\sum_{n=0}^{\infty} a_{n} \cos n \pi t / \delta$. Then by (14) and (15), it can be proved that $a_{n}=O\left(1 / n^{2}\right)$. Hence $G^{\prime} \in L^{2}[-\delta, \delta]$. By (10), (11) and (13), it can also be proved that $\tilde{s}_{m}(G)=s_{m}(F ;-\delta, \delta), m=1,2, \ldots$, where $\tilde{s}_{m}(G)$ is as defined in Corollary 1 of $\S 2$, and

$$
\begin{aligned}
F\left(e^{i 2 \pi t}\right) & =f\left(e^{i 2 \pi t}\right), & & 0 \leq t \leq \delta, \\
& =f\left(e^{-i 2 \pi t}\right), & & -\delta \leq t \leq 0 .
\end{aligned}
$$

By Corollary 1 of $\$ 2$, we can conclude that $f\left(e^{i 2 \pi t}\right)=G(t)$ for $0 \leq t \leq \delta$. Therefore, we can apply a result of Patil [6] to obtain

$$
f(z)=\lim _{\lambda \rightarrow \infty} \lambda b_{\lambda}(z) \int_{0}^{\delta} \frac{\bar{b}_{\lambda}\left(e^{i 2 \pi t}\right) G(t)}{1-z e^{-i 2 \pi t}} d t
$$

for $z$ in $U$, where $G(t)$ is the limit of the uniformly convergent series (16).

Acknowledgment. We like to thank the referee for his helpful comments.

\section{REFERENCES}

1. C. H. Ching and C. K. Chui, Uniqueness theorems determined by function values at the roots of unity, J. Approximation Theory (to appear).

2. - Mean boundary value problems and Riemann series, J. Approximation Theory (to appear).

3. - Recapturing a holomorphic function on an annulus from its mean boundary values, Proc. Amer. Math. Soc. 39 (1973), 120-126.

4. G. H. Hardy and E. M. Wright, An introduction to the theory of numbers, 3rd ed., Clarendon Press, Oxford, 1954. MR 16, 673.

5. E. Landau, Elementare Zahlentheorie, Teubner, Leipzig, 1927; English transl., Chelsea, New York, 1958. MR 19, 1159.

6. D. J. Patil, Representation of $H^{p}$ functions, Bull. Amer. Math. Soc. 78 (1972), 617-620.

7. A. Zygmund, Trigonometrical series. Vol. II, 2nd rev. ed., Cambridge Univ. Press, New York, 1959. MR 21 \#6498.

DEPARTMENT OF MATHEMATICS, TEXAS A\&M UNIVERSITY, COLLEGE STATION, TEXAS 77843 (Current address of C. K. Chui)

Current address (C.-H. Ching): Department of Mathematics, University of Melbourne, Parkville, Victoria, Australia 